\title{
A Case of Gas-Forming Liver Abscess with Diabetes Mellitus
}

\author{
Tetsuya Tatsuta ${ }^{1}$, Toyohito Wada $^{2}$, Daisuke Chinda ${ }^{1}$, Kiyoto Tsushima ${ }^{1}$, Yoshio Sasaki ${ }^{2}$, \\ Tadashi Shimoyama ${ }^{1}$ and Shinsaku Fukuda ${ }^{1}$
}

\begin{abstract}
A 43-year-old man was admitted to our hospital with right hypochondriac and epigastric pain. An abdominal radiograph showed a large niveau in the right subphrenic space. An abdominal CT scan demonstrated a large liver abscess (diameter, $13 \mathrm{~cm}$ ) with gas formation. Klebsiella pneumoniae cells were isolated from the abscess, and the patient was treated with antibiotics and percutaneous drainage. It is very important to treat gas-forming liver abscess immediately, because subsequent bacteremia and septic shock are frequently noted, and the associated mortality rate is high.
\end{abstract}

Key words: Gas-forming pyogenic liver abscess (GFPLA), diabetes mellitus, Klebsiella pneumoniae

(Intern Med 50: 2329-2332, 2011)

(DOI: 10.2169/internalmedicine.50.5686)

\section{Introduction}

Gas-forming pyogenic liver abscess (GFPLA) accounts for $7 \%$ to $32 \%$ of pyogenic liver abscess (PLA) cases (1). GFPLA is commonly associated with underlying diabetes mellitus (DM). Klebsiella pneumoniae (K. pneumoniae) is the most frequent causative bacteria cultured from blood culture and liver aspirates (1). It is very important to treat GFPLA immediately, because bacteremia and septic shock frequently occur in GFPLA patients and the mortality rate is high, which accounts for $27.7 \%$ to $37.1 \%$ (1). We report a case of GFPLA with poorly controlled DM who was treated immediately without complications such as endophthalmitis or meningitis.

\section{Case Report}

A 43-year-old man was admitted to our hospital with right hypochondriac and epigastric pain for several days. His abdomen was soft, and right hypochondriac and epigastric tenderness was observed. Laboratory data at admission were as follows: aspartate aminotransferase (AST), $149 \mathrm{IU} / \mathrm{L}$ (normal range 8-38); alanine aminotransferase (ALT), 171
IU/L (normal range 4-44); alkaline phosphatase, $602 \mathrm{IU} / \mathrm{L}$ (normal range 104-338); $\gamma$-glutamyl transpeptidase, $104 \mathrm{IU} /$ L (normal range 8-60); white blood cell count, $19,820 / \mathrm{mm}^{3}$ (normal range 4,000-8,000); C-reactive protein, $26.39 \mathrm{mg} / \mathrm{dL}$ (normal range $<0.3$ ); fasting blood glucose level, $406 \mathrm{mg} / \mathrm{dL}$ (normal range 70-110); hemoglobin A1c, 14.3\% (normal range 4.3-5.8). He had taken $25 \mathrm{~g} / \mathrm{day}$ of alcohol for 23 years. There was no history of chronic obstructive pulmonary disease or glucocorticoid therapy.

A chest radiograph showed a large niveau in the right subphrenic space (Fig. 1). Ultrasonography (US) showed an arch-shaped high echoic lesion with an acoustic shadow (Fig. 2). Abdominal computed tomography (CT) scan demonstrated a large liver abscess with gas formation, and diameter of the abscess was measured as $13 \mathrm{~cm}$ (Fig. 3a). We diagnosed the patient with GFPLA and admitted him to our hospital on the same day.

We performed percutaneous transhepatic abscess drainage (PTAD) with a 7 French (Fr) pig tail tube, and $40 \mathrm{~mL}$ of brown pus and a large quantity of air were excreted. We started the treatment with broad spectrum intravenous antibiotics (1 g/day meropenem) and intravenous insulin.

Abdominal CT performed on the day after admission demonstrated that the diameter of the abscess had become

${ }^{1}$ Department of Gastroenterology, Hirosaki University Graduate School of Medicine, Japan and ${ }^{2}$ The Third Department of Internal Medicine, Aomori City Hospital, Japan

Received for publication April 21, 2011; Accepted for publication July 1, 2011

Correspondence to Dr. Tadashi Shimoyama, tsimo-hki@umin.ac.jp 

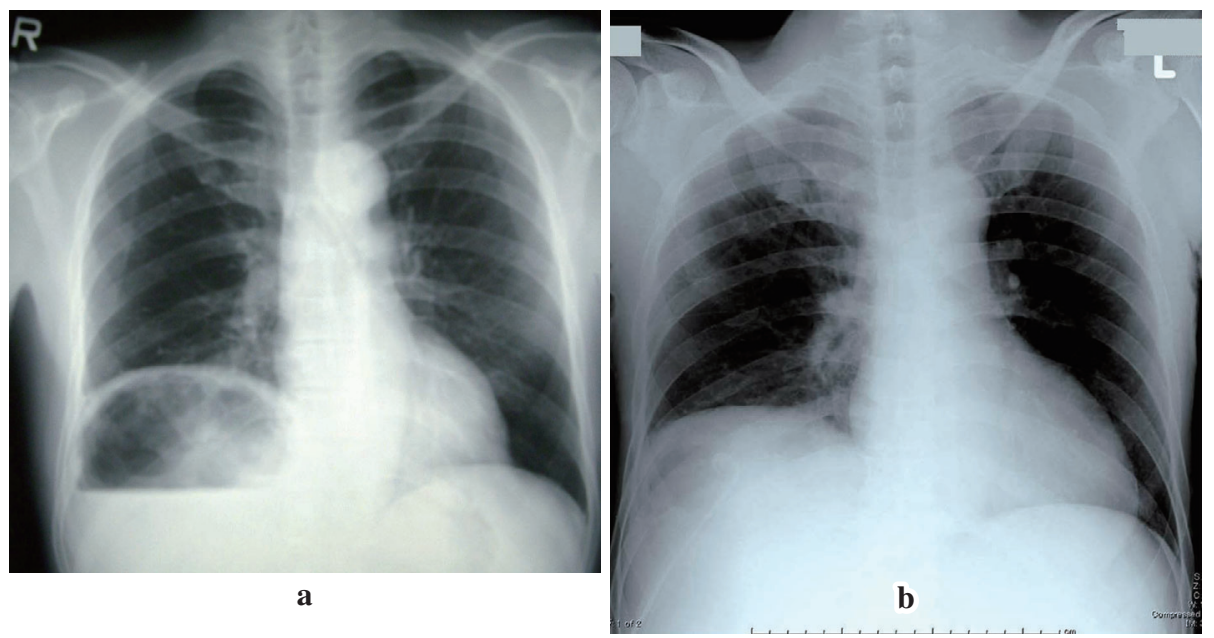

Figure 1. a: Chest X-ray film on admission shows a large niveau in the right subphrenic space. b: The niveau disappeared after PTAD.

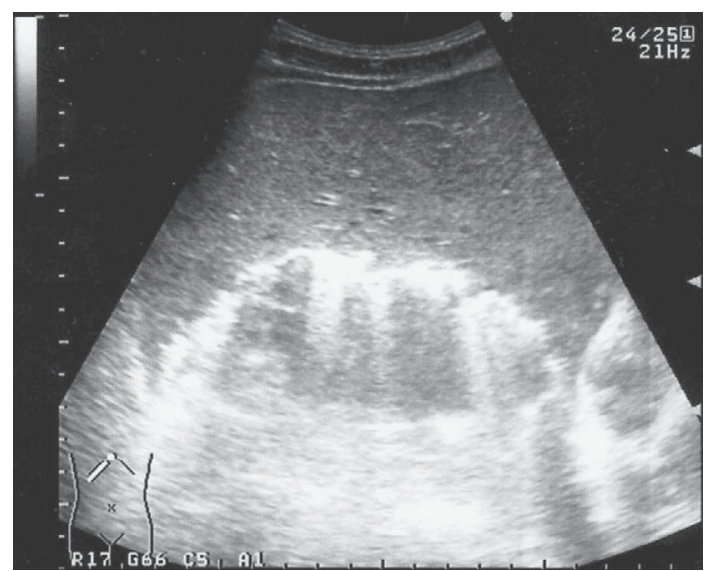

Figure 2. Ultrasonography on admission shows an archshaped high echoic lesion with an acoustic shadow.

reduced to $10 \mathrm{~cm}$, and fever and abdominal pain were improved. K. pneumoniae was cultured from pus and blood: the culture showed a high sensitivity to meropenem. Thus, the treatment with intravenous meropenem was continued. At 6 days after admission, abdominal $\mathrm{CT}$ revealed that the size of the abscess did not change even though it was wrapped by the capsule. Therefore, we replaced the 7-Fr pig tail tube with an 8-Fr tube with side holes.

Because the abscess was gradually reduced, we changed the antibiotics to oral administration of ciprofloxacin 400 $\mathrm{mg} / \mathrm{day}$ at 15 days after admission. Disappearance of the niveau was confirmed by chest radiograph (Fig. 1b). An abdominal CT on performed on day 29 revealed that the gas in the abscess had disappeared, and the diameter of the abscess was reduced to $4 \mathrm{~cm}$. The tube was removed at 34 days after admission. On the day 43 , the size of the abscess was reduced to $3 \mathrm{~cm}$ (Fig. $3 \mathrm{~b}$ ), and the patient was discharged the next day. Administration of the antibiotic was stopped on day 65 because the abscess was no longer visible on abdominal CT. The blood glucose level has been well-controlled during the treatment period, and there has a

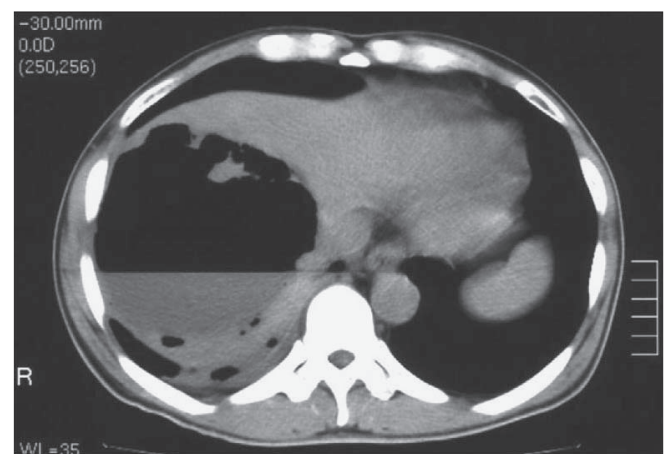

b

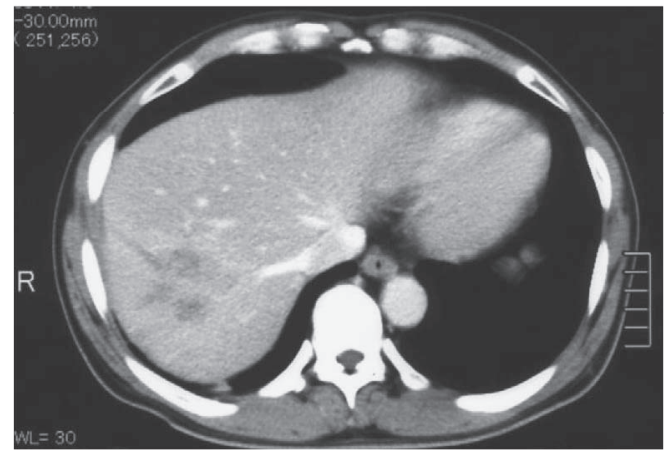

Figure 3. a: Abdominal computed tomography (CT) on admission shows a large liver abscess with gas formation, and the abscess was measured as $13 \mathrm{~cm}$ in diameter. b: Abdominal CT on $\mathbf{4 3}$ days after admission revealed that gas in the abscess disappeared and the size of the abscess was reduced to $3 \mathrm{~cm}$ in diameter.

been no clinical evidence of recurrence. Although GFPLA has been associated with the risk of digestive malignancies, esophago-gastro-duodenoscopy performed on day 31 revealed no malignancy as well as repeated abdominal US and CT. Total colonoscopy was not performed because fecal occult blood test and abdominal CT yielded negative results.

\section{Discussion}

PLA is an uncommon disease, accounting for 8 to 25 
Table 1. Laboratory Data on Admission

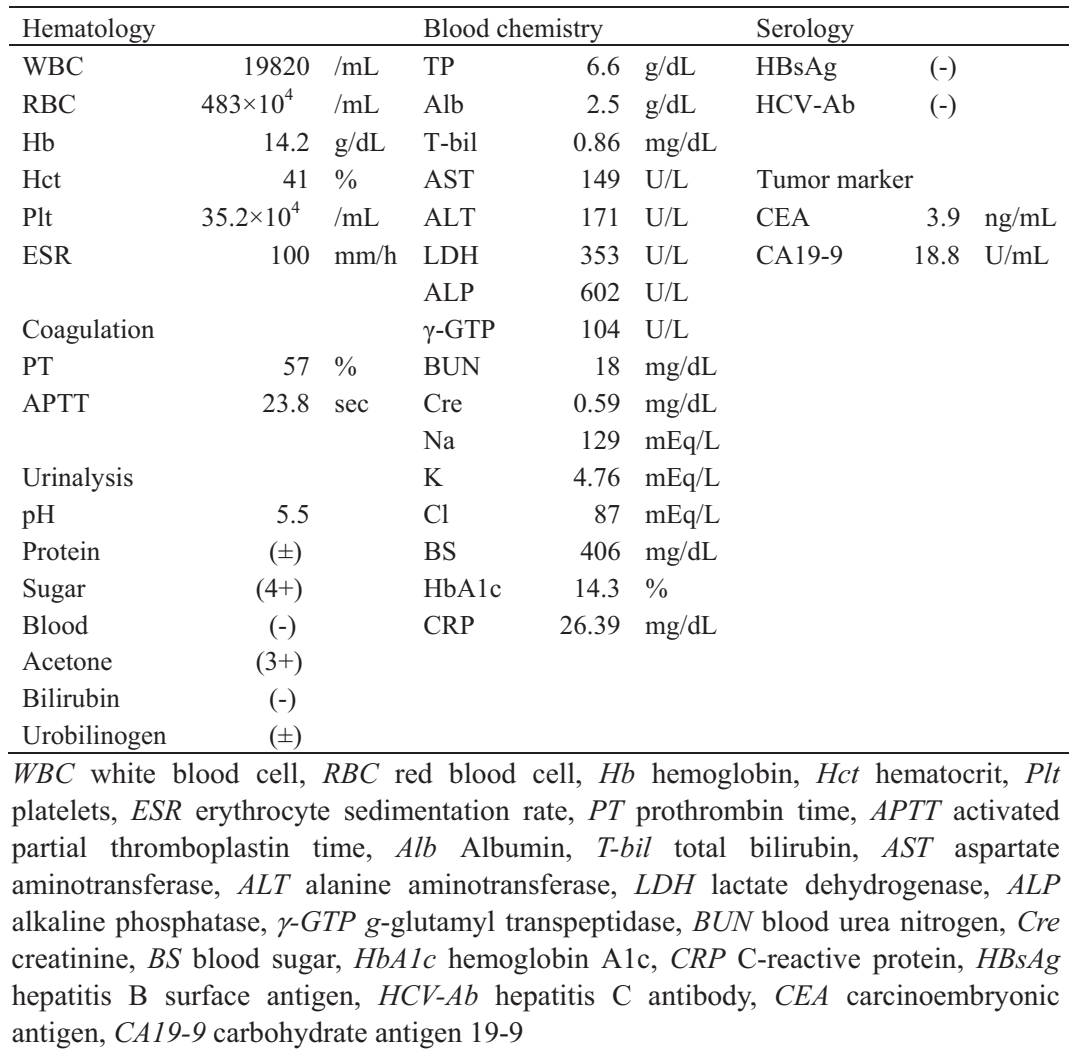

cases per 100,000 hospital admissions (1). PLA is divided into 7 groups on the basis of the infection route: portal vein route, liver artery, biliary tract, direct route, injury, iatrogenic, and idiopathic routes. Among these, the biliary tract route is the most frequent route, $60 \%$ of PLA cases occur via biliary tract (2). Ultrasonography has been successful for the diagnosis of PLA in 95\% of the cases, and almost all the cases can be diagnosed by CT (2-4). GFPLA accounts for $7 \%$ to $32 \%$ of PLA cases. It has been reported that of the 69 patients who had PLA, 22 had GFPLA, and 21 had DM (1). K. pneumoniae is the most common pathogen of PLA, which accounts for $70 \%$ of GFPLA (1).

Hyperglycemia is an important factor for GFPLA. Poor control of DM leads to compromised immunity, and neutrophil dysfunction and chemotaxis dysfunction. These provide a favorable microenvironment for the rapid growth and vigorous metabolism of the bacteria, which in turn may lead to gas production (5). Local tissue damage caused by gasforming bacteria and compounded by the diabetic microangiopathy would markedly retard the transport of catabolic end products away from the lesion and thereby results in gas accumulation (1). The present patient also had poorlycontrolled DM, which would play a role in the development of GFPLA.

Metastatic infection including endophthalmitis and meningitis are more frequent in the K. pneumoniae GFPLA than in the non-K. pneumoniae GFPLA (6). Because two-thirds of PLA patients had permanent vision loss, physicians should consult an ophthalmologist rapidly whenever a diag- nosis of PLA caused by K. pneumoniae is made. In the present case, we consulted an ophthalmologist on the day after admission, and the patient had no endophthalmitis or DMassociated retinopathy.

Among patients with PLA, the incidence of septic shock and bacteremia is higher in GFPLA patients compared to non-GFPLA patients. Septic shock is noted in $32.5 \%$ of patients with GFPLA and in $11.7 \%$ of patients with PLA patients (7). GFPLA also has a high fatality rate which is around $27.7 \%$ to $37.1 \%$ (1). GFPLA also ruptures easily because of tissue invasion and fragility of abscess wall, and further gas formation increases the internal pressure of the abscess (8). In previous studies, spontaneous rupture of PLA had occurred in $7.1 \%$ to $15.1 \%$ of the cases (9) and 20 of 24 patients with ruptured PLA had GFPLA (8). To treat GFPLA, PTAD is usually performed to evacuate the purulent material inside the abscess if the abscess size is larger than $5 \mathrm{~cm}$ along with the administration of intravenous antibiotics (2). Indications for the removal of the tube are negative culture results, and a daily drainage output of less than $5 \mathrm{~mL}$ (6). Although some large abscesses do not disappear completely, the risk of recurrence is very low if the tube is removed after the improvement in inflammation. In the present case, we removed the tube when the diameter of the abscess on the scan was $4 \mathrm{~cm}$ and no recurrence was observed to date.

We should select antibiotics to target gram-negative bacilli such as K. pneumoniae and E. coli. Because treatment results were similar between patients treated with extended- 
spectrum cephalosporins and those treated with a combination of first-generation cephalosporins and aminoglycosides, treatment with the latter is recommended for patients without risk factors such as endophthalmitis and meningitis (10).

In conclusion, we report a case of GFPLA who was treated immediately with PTAD and intravenous administration of an antibiotic and had a good course without complications such as endophthalmitis and/or meningitis.

The authors state that they have no Conflict of Interest (COI).

\section{References}

1. Lee HL, Lee HC, Guo HR, Ko WC, Chen KW. Clinical significance and mechanism of gas formation of pyogenic liver abscess due to Klebsiella pneumoniae. J Clin Microbiol 42: 2783-2785, 2004.

2. Wong WM, Wong BC, Hui CK, et al. Pyogenic liver abscess: Retrospective analysis of 80 cases over a 10-year period. J Gastroenterol Hepatol 17: 1001-1007, 2002.

3. Mohsen AH, Green ST, Read RC, McKendrick MW. Liver abscess in adults: ten years experience in a UK centre. Q J Med 95: 797802, 2002.

4. Kaplan GG, Gregson DB, Laupland KB. Population-based study of the epidemiology of and the risk factors for pyogenic liver abscess. Clin Gastroenterol Hepatol 2: 1032-1038, 2004.

5. Chong VH, Yong A, Wahab AY. Gas-forming pyogenic liver abscess. Singapore Med J 49: 123-125, 2008.

6. Yang $\mathrm{CC}$, Yen $\mathrm{CH}$, Ho MW, Wang JH. Comparison of pyogenic liver abscess caused by non-Klebsiella pneumoniae and Klebsiella pneumoniae. J Microbiol Immunol Infect 37: 176-184, 2004.

7. Chou FF, Sheen-Chen SC, Chen YS, Lee TY. The comparison of clinical course and results of treatment between gas-forming and non-gas-forming pyogenic liver abscess. Arch Surg 130: 401-406, 1995.

8. Maeda M, Miyashita T. A case of a gas-containing liver abscess into the peritoneal cavity. J Jpn Surg Assoc 67: 861-864, 2006 (in Japanese with English abstract).

9. Siu WT, Chan WC, Hou SM, Li MK. Laparoscopic management of ruptured pyogenic liver abscess. Surg Laparosc Endosc 7: 426428, 1997.

10. Lee SS, Chen YS, Tsai HC, et al. Predictors of septic metastatic infection and mortality among patients with Klebsiella pneumoniae liver abscess. Clin Infect Dis 47: 642-650, 2008.

(C) 2011 The Japanese Society of Internal Medicine http://www.naika.or.jp/imindex.html 